\title{
ANÁLISE DO POTENCIAL MUTAGÊNICO DOS ESTEROIDES ANABÓLICOS ANDROGÊNICOS (EAA) E DA L-CARNITINA MEDIANTE O TESTE DO MICRONÚCLEO EM ERITRÓCITOS POLICROMÁTICOS
}

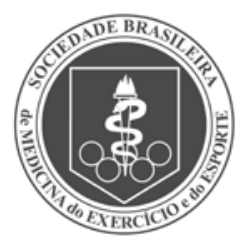

Artigo Original

\author{
ANALYSIS OF MUTAGENIC POTENTIAL OF THE ANABOLIC ANDROGENIC STEROIDS (AAS) AND L-CARNITINE \\ BY THE MICRONUCLEUS TEST IN POLYCHROMATIC ERYTHROCYTES
}

\begin{abstract}
ANÁLISIS DEL POTENCIAL MUTAGÉNICO DE LOS ESTEROIDES ANABÓLICOS ANDROGÉNICOS (EAA) Y DE LA L-CARNITINA MEDIANTE LA PRUEBA DEL MICRONÚCLEO EN ERITROCITOS POLICROMÁTICOS
\end{abstract}

\author{
Rodrigo Pinheiro Araldi (Biólogo)' \\ Décio Gomes de Oliveira \\ (Biólogo) $)^{2}$ \\ Douglas Fernandes da Silva \\ (Biólogo) ${ }^{1}$ \\ Thais Biude Mendes (Biólogo) ${ }^{1}$ \\ Edislane Barreiros de Souza \\ (Biólogo) $)^{1}$

\begin{abstract}
1. UNESP - Universidade Estadual Paulista "Júlio de Mesquita Filho", Faculdade de Ciências e Letras de Assis (FCL), Departamento de Ciências Biológicas, Laboratório de Biologia Molecular, Genética e Mutagênese - Assis, SP, Brasil. 2. Unoeste - Universidade do Oeste Paulista - Presidente Prudente, SP, Brasil.
\end{abstract}

Correspondência: Edislane Barreiros de Souza Av. Dom Antônio, 2.100, Parque Universitário. 19806-900 - Assis, SP, Brasil edislane@assis.unesp.br

\section{RESUMO}

Introdução: Os esteroides anabólicos androgênicos são usados por pessoas que desejam aumentar sua massa muscular para obter um melhor desempenho nos esportes ou melhorar a aparência física. Os EAA são derivados sintéticos da testosterona, capazes de promover a hipertrofia das fibras musculares, aumentando a síntese proteica intracelular. A L-carnitina é um suplemento alimentar empregado para aumentar a produção energética por meio da oxidação de ácidos graxos. Embora haja trabalhos mostrando as propriedades fisiológicas dessas drogas, há poucos estudos sobre o potencial mutagênico das mesmas. Objetivos: Este trabalho avaliou a clastogenicidade e genotoxicidade do decanoato de nandrolona, decanoato de testosterona e da L-carnitina, em diferentes tratamentos, através do teste do micronúcleo em eritrócitos policromáticos de ratos Wistar. Métodos: Os animais foram submetidos a diferentes concentrações e associações de EAA. O controle positivo recebeu ciclofosfamida $50 \mathrm{mg} / \mathrm{kg}$ através de injeção intraperitoneal e o controle negativo, $1 \mathrm{ml}$ de soro fisiológico por gavagem. Os ratos foram sacrificados após 36 horas da última aplicação, tendo seus fêmures removidos e a medula óssea extraída. O material foi homogeneizado e centrifugado. O botão de células foi pipetado e transferido para as lâminas, que foram coradas com Giemsa. Foram contados 1.000 eritrócitos policromáticos por animal, observando a frequência de micronúcleos. Resultados: Foi realizado o teste de Kruskal-Wallis, com nível de significância de 5\%, que demostrou que o decanoato de nandrolona três doses de 0,2 mg/kg e 0,6 mg/ $/ \mathrm{kg}$, oito doses de 7,5 mg/kg, L-carnitina - sete doses de 0,4 ml/250 e 1,5 $\mathrm{ml} / 250 \mathrm{~g}$, decanoato de testosterona - 28 doses de $0,075 \mathrm{mg} / \mathrm{kg}$, decanoato de nandrolona - oito doses de $7,5 \mathrm{mg} / \mathrm{kg}$ associado a L-carnitina $1 \mathrm{ml}$ e decanoato de nandrolona - oito doses de 7,5 mg/ $\mathrm{kg}$ associado à decanoato de testosterona - oito doses de $7,5 \mathrm{mg} / \mathrm{kg}$ apresentaram potencial mutagênico. Conclusão: Os tratamentos revelaram-se clastogênicos, não sendo indicado como recurso ergogênico.

Palavras-chave: anabolizantes, suplementos dietéticos, acetilcarnitina, genotoxicidade.

\begin{abstract}
Introduction: Anabolic androgenic steroids (AAS) are frequently used by people whose aim to increase muscle mass to obtain a better performance in sports or improve physical appearance. AAS are synthetic derivatives of testosterone, able to promote muscle fibers hypertrophy, increasing intracellular protein synthesis. L-carnitine is a food supplement used to increase energetic production by means of fat acids oxidation. Although there are several works about physiological properties of these drugs, there are few studies about their mutagenic potential. Objectives: This work evaluated the clastogenicity and genotoxicity of nandrolone decanoate, testosterone decanoate and L-carnitine, in different treatments through the micronucleus test in polychromatic erythrocytes of Wistar rats. Methods: The animals were submitted to different concentrations and associations of AAS. The positive control received cyclophosphamide $50 \mathrm{mg} / \mathrm{kg}$ by intraperitoneal injection and negative control, one $\mathrm{ml}$ of saline solution by gavage. The rats were sacrificed after 36 hours of latest application, having the femurs removed and the bone marrow extracted. Material was homogenized and centrifuged. Button cell was pipetted and transferred to slides, which were stained by Giemsa. 1,000 polychromatic erythrocytes were counted per animal, noting the frequency of micronuclei. Results: The Kruskal-Wallis test was performed, with a significance level of 5\%, which demonstrated that nandrolone decanoate - three doses of $0,2 \mathrm{mg} / \mathrm{kg}$ and $0,6 \mathrm{mg} / \mathrm{kg}$, eight doses of $7,5 \mathrm{mg} / \mathrm{kg}$, L-carnitine - seven doses of 0,4 m//250 g and 1,5 m//250 g, testosterone decanoate - 28 doses of 0,075 mg/kg, nandrolone decanoate - eight doses of 7,5 mg/kg associated to L-carnitine and $1 \mathrm{~mL}$ and nandrolone decanoate - eight doses of 7,5 mg/ $\mathrm{kg}$ associated to testosterone decanoate - eight doses of 7,5 mg/kg, showed mutagenic potential. Conclusion: The treatments proved to be clastogenic, not being indicated like ergogenic aid.
\end{abstract}

Keywords: anabolic agents, dietary supplements, acetylcarnitine, genotoxicity. 


\section{RESUMEM}

Introducción: Los esteroides anabólicos androgénicos son usados por personas que quieren aumentar su masa muscular a fin de obtener un mejor desempeño en los deportes o para mejorar la apariencia física. Los EAA son derivados sintéticos de la testosterona, que pueden causar la hipertrofia de las fibras musculares, aumentando la sintesis proteica intracelular. La L-carnitina es un suplemento alimenticio utilizado para hacer crecer la producción energética por medio de la oxidación de ácidos grasos. Aunque hay trabajos que muestran las propiedades fisiológicas de esos medicamentos, hay pocos estudios sobre el potencial mutagénico de estos. Objetivos: Este trabajo evaluó la clastogenicidad l la genotoxicidad del decanoato de nandrolona, decanoato de testosterona y de la L-carnitina, en diversos tratamientos, mediante la prueba del micronúcleo en eritrocitos policromáticos de ratones Wistar. Métodos: Los animales fueron sometidos a diferentes concentraciones y asociaciones de EAA. El control positivo recibió ciclofosfamida $50 \mathrm{mg} / \mathrm{kg}$ a través de inyección intraperitoneal, y el control negativo $1 \mathrm{ml}$ de suero fisiológico por gavaje. Los ratones fueron sacrificados después de 36 horas de la última aplicación, teniendo sus fémures removidos y la médula ósea extraída. El material fue homogeneizado y centrifugado. El botón de células fue tomado con pipetas y transferido para las láminas, a las cuales se les dio color con Giemsa. Fueron contados 1.000 eritrocitos policromáticos por animal, observándose la frecuencia de micronúcleos. Resultados: Se realizó la prueba de Kruskal-Wallis, con nivel de significancia de 5\%, la cual demostró que el decanoato de nandrolona - tres dosis de 0,2 mg/kg y 0,6 mg/kg, ocho dosis de 7,5 mg/kg, L-carnitina - siete dosis de 0,4 m/ $/ 250 \mathrm{~g}$ y 1,5 m/ $/ 250 \mathrm{~g}$, decanoato de testosterona - 28 dosis de 0,075 $\mathrm{mg} / \mathrm{kg}$, decanoato de nandrolona - ocho dosis de $7,5 \mathrm{mg} / \mathrm{kg}$ asociado a L-carnitina $1 \mathrm{mly}$ decanoato de nandrolona - ocho dosis de 7,5 mg/kg vinculado al decanoato de testosterona - ocho dosis de 7,5 mg/kg, presentaron potencial mutagénico. Conclusión: Los tratamientos se revelaron como siendo clastogénicos, no indicándose como recurso ergogénico.

Palabras clave: anabolizantes, suplementos dietéticos, acetilcarnitina, genotoxicidad.

Artigo recebido em 01/04/2011, aprovado em 07/10/2013.

\section{INTRODUÇÃO}

Os esteroides anabólicos androgênicos (EAA) são derivados sintéticos do hormônio testosterona, sendo agentes farmacológicos frequentemente empregados como recursos ergogênicos, agindo da mesma forma que os androgênicos. Os EAA são usados para promover aumento da massa muscular, com o objetivo de obter um melhor desempenho nos esportes e melhorar a aparência física ${ }^{1}$. Entretanto, as propriedades androgênicas são responsáveis por diversos efeitos colaterais².

O efeito anabólico é resultado da propriedade da testosterona em promover a hipertrofia das fibras musculares, devido ao aumento da síntese proteica intracelular, levando a um aumento da massa muscular. Os esteroides sintéticos promovem o aumento da contratilidade muscular e o aumento do volume celular dos miócitos através dos seguintes mecanismos: promoção da estocagem de fósforo-creatina, acúmulo de glicogênio, favorecimento da recaptação de aminoácidos e bloqueio do cortisol ${ }^{3}$. Por outro lado, os efeitos da testosterona podem levar ao desenvolvimento de características sexuais masculinas secundárias como crescimento do escroto e do pênis, surgimento de pelos púbicos, axilares e faciais, desenvolvimento da laringe, tornando as cordas vocais mais grossas, resultando em voz com timbre baixo, aumento da atividade das glândulas sebáceas, espessamento da pele e alterações psicológicas ${ }^{3-5}$.

Os EAA são frequentemente utilizados em combinação com programas de treinamento e ingestão de proteínas, a fim de melhorar o desempenho em esportes que demandam força e velocidade ${ }^{6}$. Os EAA estão, também, associados ao consumo abusivo de álcool, morfina e outras substâncias ativas. Nos Estados Unidos e Canadá, um a cada cinco adolescentes faz uso de $\mathrm{EAA}^{7,8}$.

Entretanto, o uso de EAA na adolescência pode impedir o desenvolvimento ósseo, causando deformação morfofisiológica no coração, fígado e rins, podendo até mesmo levar ao óbito ${ }^{9,10}$. Os EAA também promovem aumento dos níveis de colesterol e triglicérides, provocando intoxicação hepática, dependência do esteroide sintético. O uso destes EAA está associado ao aumento da incidência de câncer hepático e hematológico ${ }^{11}$.

Os EAA representam um grande número de substâncias lipofílicas que têm a estrutura básica do colesterol, molécula precursora de todos os esteroides. Este grupo de substâncias inclui estrógenos, corticosteroi- des, progesterona, mineralocorticoides e andrógenos ${ }^{12,13}$. Os esteroides variam em estrutura, número, posição de grupos funcionais, cadeias laterais ligadas ao núcleo e outras características químicas ${ }^{13,14}$.

A partir da molécula de testosterona natural, são sintetizadas outras substâncias, resultando em um grupo de drogas denominadas de esteroides anabólicos androgênicos ${ }^{14,15}$.

Os hormônios esteroidais apresentam quatro mecanismos básicos de ação: aumento da síntese proteica da musculatura esquelética, redução do processo catabólico, atuação sob o sistema nervoso central e efeito placebo ${ }^{16,17}$. Um dos EAA mais usados atualmente é o Durateston ${ }^{\circledR}$, sendo facilmente encontrado no mercado.

O Durateston ${ }^{\circledR}$ é um EAA injetável, comercializado em ampolas contendo $250 \mathrm{mg} / \mathrm{ml}$, produzido no Brasil pela Organon, contendo 30 $\mathrm{mg}$ de propionato de testosterona, $60 \mathrm{mg}$ de fenilpropionato de testosterona, $60 \mathrm{mg}$ de isocaproato de testosterona, $100 \mathrm{mg}$ de caproato de testosterona e $1 \mathrm{ml}$ de óleo de amendoim.

Atualmente, suplementos alimentares e esteroides anabólicos são muito utilizados por praticantes de atividades físicas de diversas modalidades ${ }^{18-24}$; embora os anabólicos sejam ilegais, eles são utilizados de forma abusiva por muitos esportistas. Há uma nova tendência no uso de suplementos alimentares como alternativa legal para promover a ativação do metabolismo anabólico ${ }^{25}$. Entre estes suplementos, a L-carnitina é o mais empregado.

A carnitina (L-trimetil-3-hidróxiamoniobutanoato) é uma amina quaternária com função de gerar energia à célula porque age na reação de transferência da cadeia longa de ácidos graxos do citosol para a mitocôndria, facilitando a oxidação e a geração de adenosina trifosfato (ATP) 26,27 .

A L-carnitina é produzida pela farmacêutica Midway International Labs Ltd., sendo constituída de L-carnitina associada à vitamina $B 5^{28}$. Além disso, a L-carnitina promove o aumento dos níveis de $\mathrm{HDL}$, que pode auxiliar na diminuição da pressão arterial ${ }^{28}$ e tem sido empregada em pacientes portadores de doenças cardiovasculares devido à promoção dos mecanismos de contratilidade das células musculares cardíacas e da regulação da níveis de ésteres de acetil-CoA no miocárdio ${ }^{29}$.

O teste do micronúcleo em eritrócitos policromáticos foi desenvolvido por Schimid et al. ${ }^{36}$ e modificado por Hedle et al., tendo sido usado pela 
primeira vez em eritrócitos da medula óssea de camundongos ${ }^{30}$. Este teste é preconizado por agências internacionais e instituições governamentais, como parte dos testes de avaliação de novos produtos químicos ou fármacos que entram no mercado ${ }^{30}$. O teste do micronúcleo consiste em uma importante técnica citogenética não invasiva que permite detectar mutações cromossômicas que são a chave para os eventos carcinogênicos ${ }^{31,32}$.

O teste pode ser usado em ecogenotoxicidade ${ }^{33}$, investigação de danos ao DNA ${ }^{31}$, quebras cromossômicas ou interferência no fuso mitótico e modificações equitativas da distribuição cromossômica durante a divisão celular ${ }^{34}$.

O termo micronúcleo foi sugerido por Boller et al. em 197035, designando corpos cromáticos com diâmetro menor que 1/3 do diâmetro do núcleo celular ${ }^{36}$, produzidos ao longo da anáfase devido aos defeitos nos centrômeros ou fragmentos cromossômicos ou cromossomos inteiros perdidos em função de danos recentemente induzidos ${ }^{31}$.

\section{MÉTODOS}

Foram utilizados ratos Wistar, com 12 semanas, provenientes do Biotério Central da UNESP - campus de Botucatu. Os ratos foram mantidos no Biotério da UNESP - FCL sob as condições padrão (ciclo claro/ escuro de 12:12 horas, temperatura de $22^{\circ} \mathrm{C} \pm 2^{\circ} \mathrm{C}$, luminosidade de 150 lux, água e ração sólida ad libitum). Os ratos foram divididos nos grupos controle positivo, negativo e experimentais. O grupo controle positivo foi tratado com ciclofosfamida (CAS 50-18-0) $50 \mathrm{mg} / \mathrm{kg}$, lote 108H0568, da Sigma-Aldrich ${ }^{\circledR}$ (St. Luis, MO, EUA), tendo recebido a droga por meio de injeção intraperitoneal. O grupo controle negativo foi tratado com $1 \mathrm{ml}$ de soro fisiológico por meio de gavagem e os grupos experimentais receberam os tratamentos mostrados na tabela 1.

Os ratos foram sacrificados após 36 horas da última gavagem. Os fêmures foram removidos e a medula óssea foi extraída injetando-se $1 \mathrm{ml}$ de soro bovino fetal (Cultilab ${ }^{\circledR}$, Campinas, SP, Brasil) com o auxílio de uma seringa com agulha. O material obtido foi homogeneizado com o auxílio de uma pipeta de Pasteur e centrifugado a 1.500 RPM em centrífuga da maraca Fanem ${ }^{\circledR}$, modelo 206BL (Guarulhos, SP), por cinco minutos. Após a centrifugação, o botão de células foi colhido e o sobrenadante foi descartado. O material foi transferido para as lâminas, previamente limpas com álcool $70^{\circ}$, as quais foram levadas a uma estufa Fanem ${ }^{\circledR}$, modelo 002C13 (Guarulhos, SP, Brasil) por duas horas para a

Tabela 1. Substância administrada por tratamento.

\begin{tabular}{|c|c|}
\hline Tratamento & Substância utilizada/dose administrada \\
\hline 1 & Decanoato de nandrolona - dose única de 0,2 mg/250 g \\
\hline 2 & Decanoato de nandrolona - dose única de 0,6 mg/250g \\
\hline 3 & Decanoato de nandrolona - três doses de $0,2 \mathrm{mg} / 250 \mathrm{~g}$ \\
\hline 4 & Decanoato de nandrolona - três doses de 0,6 mg/250g \\
\hline 5 & Decanoato de nandrolona - oito doses de 7,5 mg/kg \\
\hline 6 & Decanoato de testosterona -28 doses de $0,075 \mathrm{mg} / \mathrm{kg}$ \\
\hline 7 & L-carnitina - sete doses de $0,4 \mathrm{ml} / 250 \mathrm{~g}$ \\
\hline 8 & L-carnitina - sete doses de $1,5 \mathrm{ml} / 250 \mathrm{~g}$ \\
\hline 9 & L-carnitina - 28 doses de $1 \mathrm{ml}$ \\
\hline 10 & $\begin{array}{l}\text { Ciclofosfamida - dose única de } 50 \text { mg/kg } \\
\text { (controle positivo, grupos } 1 \text { ao 9) }\end{array}$ \\
\hline 11 & $\begin{array}{l}\text { Solução fisiológica - dose única de } 1 \mathrm{ml} \\
\text { (controle negativo, grupos } 1 \text { ao 9) }\end{array}$ \\
\hline 12 & $\begin{array}{c}\text { Decanoato de nandrolona - oito doses de } 7,5 \mathrm{mg} / \mathrm{kg} \text { associado à } \\
\text { Decanoato de testosterona - oito doses de 7,5 mg/kg, com } \\
\text { intervalo de sete dias entre as aplicações }\end{array}$ \\
\hline 13 & $\begin{array}{c}\text { Decanoato de nandrolona - oito doses de } 7,5 \mathrm{mg} / \mathrm{kg} \text { associadas } \\
\text { a L-carnitina }-1 \mathrm{ml} \text {, com intervalo de sete dias }\end{array}$ \\
\hline 14 & $\begin{array}{c}\text { Decanoato de testosterona - } 28 \text { doses de } 0,075 \mathrm{mg} / \mathrm{kg} \text { associadas } \\
\text { a L-carnitina }-1 \mathrm{ml} \text {, com intervalo de } 24 \text { horas }\end{array}$ \\
\hline 15 & $\begin{array}{c}\text { Ciclofosfamida - dose única de } 50 \mathrm{mg} / \mathrm{kg} \\
\text { (controle positivo, grupos } 12 \text { ao 14) }\end{array}$ \\
\hline 16 & $\begin{array}{l}\text { Solução fisiológica - dose única de } 1 \mathrm{ml} \\
\text { (controle negativo, grupos } 12 \text { ao 14) }\end{array}$ \\
\hline
\end{tabular}

secagem do material. O material foi fixado em etanol 100\% por cinco minutos e, após a secagem, foi corado de com azul-eosina metileno (Giemsa) de acordo com as seguintes etapas: três minutos em Giemsa absoluto, lavagem em água destilada, um minuto em solução de 1:5 Giemsa-tampão fosfato pH 6,0 e lavagem em xilol.

As lâminas foram montadas e analisadas no microscópio óptico binocular Olympus ${ }^{\circledR}$ Cx41 (Center Valley, PA, EUA), tendo sido contada a frequência de eritrócitos policromáticos micronucleados (EPCMN) a cada 2.000 eritrócitos policromáticos por animal, de acordo com o protocolo padronizado para o teste.

A análise estatística foi realizada por meio de uma análise de variância não paramétrica, através do teste de Kruskal-Wallis, seguido do teste post hoc de Dunn, ambos com nível de significância de 5\%, através do software Statistic versão 7.0.

\section{RESULTADOS}

A fim de comparar a frequência de EPCMN entre os tratamentos, foi realizado o teste de Kruskal-Wallis (tabela 2). O teste mostrou haver diferença estatística entre os protocolos $(H=131,048$ e $p=0,000)$ (tabela 3).

Tabela 2. Frequência de eritrócitos policromáticos micronucleados (EPCMN) observada por animal, seguido do número total de EPCMNF por protocolo.

\begin{tabular}{c|c|c|c|c|c|c|c|c|c|c|c}
\hline Protocolo & $\mathbf{1}$ & $\mathbf{2}$ & $\mathbf{3}$ & $\mathbf{4}$ & $\mathbf{5}$ & $\mathbf{6}$ & $\mathbf{7}$ & $\mathbf{8}$ & $\mathbf{9}$ & $\mathbf{1 0}$ & Total \\
\hline 1 & 04 & 02 & 03 & 03 & 05 & 02 & 06 & 03 & 04 & 05 & 37 \\
\hline 2 & 20 & 13 & 21 & 24 & 22 & 25 & 18 & 23 & 22 & 24 & 212 \\
\hline 3 & 60 & 61 & 52 & 68 & 62 & 56 & 63 & 62 & 72 & 65 & 621 \\
\hline 4 & 102 & 110 & 108 & 128 & 126 & 131 & 112 & 115 & 121 & 136 & 1189 \\
\hline 5 & 08 & 08 & 05 & 05 & 08 & 03 & 08 & 07 & 06 & 07 & 65 \\
\hline 6 & 19 & 09 & 10 & 06 & 05 & 08 & 08 & 07 & 09 & 08 & 89 \\
\hline 7 & 06 & 06 & 08 & 05 & 07 & 06 & 06 & 09 & 05 & 07 & 65 \\
\hline 8 & 13 & 08 & 09 & 05 & 06 & 09 & 05 & 07 & 05 & 06 & 73 \\
\hline 9 & 05 & 04 & 04 & 03 & 01 & 02 & 03 & 02 & 02 & 01 & 27 \\
\hline 10 & 20 & 22 & 29 & 26 & 31 & 42 & 40 & 38 & 45 & 48 & 343 \\
\hline 11 & 00 & 02 & 01 & 03 & 02 & 05 & 03 & 02 & 02 & 03 & 23 \\
\hline 12 & 04 & 02 & 03 & 03 & 05 & 02 & 06 & 03 & 04 & 05 & 72 \\
\hline 13 & 20 & 13 & 21 & 24 & 22 & 25 & 18 & 23 & 22 & 24 & 93 \\
\hline 14 & 60 & 61 & 52 & 68 & 62 & 56 & 63 & 62 & 72 & 65 & 82 \\
\hline 15 & 102 & 110 & 108 & 128 & 126 & 131 & 112 & 115 & 121 & 136 & 65 \\
\hline 16 & 08 & 08 & 05 & 05 & 08 & 03 & 08 & 07 & 06 & 07 & 32 \\
\hline
\end{tabular}

Tabela 3. Comparação da frequência de EPCMN por protocolo através do teste post hoc de Dunn.

\begin{tabular}{c|c|c}
\hline Tratamento & Mediana & Desvio interquartílico \\
\hline 4 & $118 \mathrm{a}^{*}$ & 17,00 \\
\hline 3 & $62 \mathrm{a}$ & 4,25 \\
\hline 10 & $34,5 \mathrm{a}$ & 14,75 \\
\hline 2 & $22 \mathrm{ab}$ & 3,50 \\
\hline 13 & $8 \mathrm{bc}$ & 3,75 \\
\hline 6 & $8 \mathrm{bc}$ & 1,75 \\
\hline 14 & $7,5 \mathrm{c}$ & 5,00 \\
\hline 8 & $6,5 \mathrm{c}$ & 3,50 \\
\hline 12 & $7 \mathrm{c}$ & 3,75 \\
\hline 5 & $7 \mathrm{~cd}$ & 2,75 \\
\hline 7 & $6 \mathrm{~cd}$ & 1,00 \\
\hline 15 & $6,5 \mathrm{ce}$ & 4,00 \\
\hline 1 & $3,5 \mathrm{df}$ & 1,75 \\
\hline 16 & $3 \mathrm{ef}$ & 0,75 \\
\hline 9 & $2,5 \mathrm{f}$ & 1,75 \\
\hline 11 & $2 \mathrm{f}$ & 1,00 \\
\hline
\end{tabular}

*letras iguais indicam não haver diferença estatística $(p<0,05)$.

\section{DISCUSSÃO}

Embora o uso dos EAA seja frequente, há poucos estudos envolvendo a avaliação do potencial clastogênico e genotóxico dessas drogas, fazendo deste um importante estudo, pois permite avaliar a segurança dessas drogas quanto a eventuais danos à molécula de DNA, os quais são a chave para carcinogênese ${ }^{31}$. 
De acordo com a tabela 2, é possível afirmar que os tratamentos 3, 4 e 10 (decanoato de nandrolona - três doses de 0,2 mg/250 g, decanoato de nandrolona - três doses de $0,6 \mathrm{mg} / 250 \mathrm{~g}$ e ciclofosfamida $50 \mathrm{mg} / \mathrm{kg}$, respectivamente) não apresentaram diferenças estatísticas significativas quanto à frequência de EPCMN, embora tenham apresentado a maior frequência de micronúcleos. Estes dados apontam para um potencial efeito mutagênico conferido pelos tratamentos. Em relação ao potencial clastogênico e genotóxico conferido pela ciclofosfamida, tratamento 10, este está em conformidade com o esperado, haja vista que a droga foi utilizada como controle positivo das condições experimentais. Os dados estão, também, em conformidade com o estudo envolvendo o decanoato de nandrolona $7,5 \mathrm{mg} / \mathrm{kg}$ em ratos Wistar durante oito semanas, que demostrou o potencial mutagênico da droga ${ }^{37}$ bem como desta associada ao suplemento alimentar L-carnitina ${ }^{38}$.

Este resultado permite pontuar os tratamentos 3 e 4 como inapropriados como recursos ergogênicos, pois podem resultar em danos ao DNA, os quais podem levar a um processo carcinogênico ${ }^{30,31}$, demostrando a relação entre o uso dos EAA e a incidência de câncer hepático e hematopoiético ${ }^{11}$.

De acordo com o teste post hoc de Dunn, os tratamentos 9 e 11 (L-carnitina - 28 doses de $1 \mathrm{ml}$ e solução fisiológica - dose única de $1 \mathrm{ml}$, respectivamente) mostraram não haver diferença estatística entre eles quanto à frequência de EPCMN, bem como não evidenciando efeitos clastogênicos e/ou genotóxicos. Este resultado está em consonância com o esperado para o tratamento 11, haja vista que o soro fisiológico foi usado como controle negativo das condições.

Com base nestes resultados, é possível assegurar que a L-Carnitine Way 1750 - 28 doses de $1 \mathrm{ml}$ não provocou danos ao DNA. Entretanto, os tratamentos com L-carnitina - sete doses de 0,4 m/ $/ 250 \mathrm{~g}$ e L-carnitina - sete doses de 1,5 ml/250 g (tratamentos 7 e 8, respectivamente) revelaram clastogenicidade e genotoxicidade. Estes dados permitem colocar

\section{REFERÊNCIAS}

1. Kindlundh AMS, Isacson DG, Berglund L. Factors associated with adolescent use of doping agents: anabolic-androgenic steroids. Addiction 1999;94:543-53.

2. Blue JG, Lombardo JA. Steroids and steroid-like compounds. Clin Sports Med 1999;18:667-89.

3. Muniz M, Afonso R, Costa VR. Anabolizantes: bombas-relógio nos músculos. Ciência Hoje 1997;22:13.

4. Cotran RS, Kumar V, Robbins S. Patologia estrutural e functional. 5a ed. Rio de Janeiro: Guanabara Koogan; 1997.

5. Guyton AC, Hall JE. Tratado de fisiologia médica. 10 ed. Rio de Janeiro: Elsevier; 2000.

6. Lovstakken K, Peterson L, Homer AL. Risk factors for anabolic steroid use in college students and the role of expectancy. Addict Behav 1999;24:425-30.

7. Stilger VG, Yesalis CE. Anabolic-androgenic steroid use among high school football players. J Com Health 1999;24:131-45.

8. Johnston LD, O'Malley PM, Bachman JG, Schulenberg JE.. Monitoring the future national results on adolescent drug use: overview of key finding. (NIH Publication No 02-2105 2002; 36). Bethsada: National Institute on Drug Abuse; 2009.

9. Brower KJ, Blow FC, Young JP, Hill EM. Symptoms and correlates of anabolic-androgenic steroid dependence. Br J Addict 1991;86:759-68.

10. Irving LM, Wall M, Neumark-Sztauner D, Story M. Steroid use among adolescents: findings from project EAT. J Adolesc Health 2002;244:243-52.

11. Yu-Yahiro JA, Michael RH, Nasrallah DV, Schofield B. Morphological and histologic abnormalities in female and male rats treated with anabolic steroid. Am J Sports Med 1989;17:686-9.

12. Stedman. Dicionário Médico. Rio de Janeiro: Guanabara Koogan; 1979.

13. Hedge GA, Colby HD, Goodman RL. Fisiologia endócrina clínica. São Paulo: Interlivros; 1973.

14. Wilson JD. Androgênios. As bases farmacológicas da terapêutica. In: Goodman G, editor. 8a ed. Rio de Janeiro: Guanabara Koogan; 1991.

15. Bagateel CJ, Bremmer WJ. Androgens in men: use and abuses. N England J 1996;334:707-13.

16. Vanburg P, Atar D. Androgenic anabolic steroid abuse and the cardiovascular system. Handb Exp Pharmacol 2010;195:411-57.

17. Blue JG, Lombardo JA. Steroids and steroid-like compounds. Clin in Sports Med 1999;61:265-72.

18. Applegate L. A mania das dietas e utilização de suplementos na pratica esportiva. Sport Science Exchange1996;4:1-4.

19. Burckley WE, Yesalis CE 3rd, Friedl KE, Anderson WA, Streit AL, et al. Estimated prevalence of anabolic steroid use among male high school senior. J American Med Assoc 1988;260:3441-5. o suplemento alimentar como não recomendado para o aumento da produção de energia, que se dá por meio da oxidação de ácidos graxos ${ }^{26}$. Os tratamentos 5, 6, 12, 13 e 15 também demostraram potencial mutagênico, fazendo dessas drogas substâncias inapropriadas como recursos ergogênicos, pois demostraram provocar danos ao material genético.

Os dados evidenciaram a frequência mais elevada de eritrócitos policromáticos micronucleados nos tratamentos com decanoato de nandrolona - três doses de 0,6 mg/250 g e decanoato de nandrolona - três doses de 0,2 mg/kg. Estas informações implicam em um elevado risco mutagênico apresentado por esses tratamentos, permitindo pontuá-los como inapropriados para o consumo como recurso ergogênico.

\section{CONCLUSÃO}

Este estudo demostrou que o decanoato de nandrolona, nos tratamentos de três doses de 0,2 mg/250g e 0,6 mg/250g, oito doses de 7,5 $\mathrm{mg} / \mathrm{Kg}$ e oito doses de $7,5 \mathrm{mg} / \mathrm{Kg}$ associado a $1 \mathrm{ml}$ de L-carnitina em intervalos de sete dias, bem como o decanoato de testosterona em 28 doses de 0,075 mg/Kg apresentaram ação clastogênica. Resultados semeIhantes foram observados no tratamento com o suplemento alimentar L-Carnitine Way 1750, em sete doses de 0,4 ml/250g e 1,5 ml/250g, o que permite concluir que os tais tratamentos promovem danos ao DNA, resultando em instabilidade genômica, podendo levar ao desenvolvimento de cânceres, contribuindo para o aumento da incidência de neoplasias hepáticas e hematológicas, associadas ao uso desses esteroides sintéticos, tornando esta uma importante preocupação de saúde, já que um a cada cinco adolescentes nos Estados Unidos e Canadá faz uso deste tipo de substância como recurso ergogênico.

Todos os autores declararam não haver qualquer potencial conflito de interesses referente a este artigo.
20. Fogelholm M. Micronutrient status in female during a 24-week fitness-type exercise program. Annals Nutr Metab 1992;36:209-18.

21. Fogelholm M, Jaakkola L, Lampisjarvi T. Effects of iron supplementation in female athletes with low serum ferritin concentration. Int J Sports Med 1992;13:158-62.

22. Fogelholm M. Indicators of vitamin and mineral status in athletes' blood: a review. Inter J Sport Nutr 1995;5:267-84.

23. Haupt HA, Rovere GD. Anabolic steroids: a review of the literature. Am J Sports Med 1984;12:469-84. 24. Moura NA. Esteróides anabólicos androgênicos e esportes: uma breve revisão. R Bras de Ciênc Esporte 1984;6:101-9.

25. McArdle WD, Katch FI, Katch VL. Fisiologia do exercício: energia, nutrição e desempenho humano. $4^{a}$ ed. Rio de Janeiro: Guanabara Koogan; 1996.

26. Cerretelli P, Marconi C. L-carnitine supplementation in humans: the effects on physical performance. Int J Sports Med 1990;11:1-14.

27. Clarkson PM. Nutritional ergogenic aids: Carnitine. Int J Sports Nutr 1992;2:185-90.

28. Eade MD. The Doctor's Complete Guide to Vitamins and Minerals. Nova York: Dell Publishing Group, 2000.

29. Lagioia R, Scruntinio D, Mangini SG, Ricci A, Mastropasqua F, Valentini G, et al. Propionyl-Lcarnitine: a new compound in the metabolic approah to the treatment of effort angina. Int J Cardiol 1992;34:167-72.

30. Ribeiro LR, Salvadori DMF, Marques EK. Mutagênese ambiental. Canoas: Ulbra, 2003.

31. Fenech M. The in vitro micronucleus technique. Mutat Res 2000;445:81-5.

32. Carbonari K, Gonçalves L, Roth D, Moreira P, Fernandez R, Martino-Roth MG. Increased micronucleated cell frequency related to exposure to radiation emitted by computer cathode ray tube video display monitors. Genet Mol Biol 2005;8:469-75.

33. Bücker A, Carvalho W, Alves-Gomes JA. Avaliação da mutagênese e genotoxicidade em Eingenmannia virescens (Teleostei: Gymnotiformes) expostos ao benzeno. Acta Amazônica 2006;36:357-64.

34. Flores M, Yamaguchi UM. Teste do Micronúcleo: uma triagem para a avaliação genotóxica. Saúde e Pesquisa 2008;1:337-40.

35. Decodier I, Kirsch-Volders M. The in vitro micronucleus test: from past to future. Mutat Res 2006;607:2-4.

36. Schimid W. The micronucleus test. Mutat Res 1976;31:9-15.

37. Oliveira DG, Kroll LB, Ronqui RA, Dias FG, Souza EB. Efeito clastogênico do Deca-Durabolin em ratos mediante a análise do micronúcleo em eritrócitos policromáticos. Plural 2000;1:91-100.

38. Tsuboy MSF, Souza EB. Avaliação mutagênica de esteroide anabólico androgênico Deca-Durabolin, pelo teste do micronúcleo in vivo [Monografia]. UNESP - FCL; 2005. 